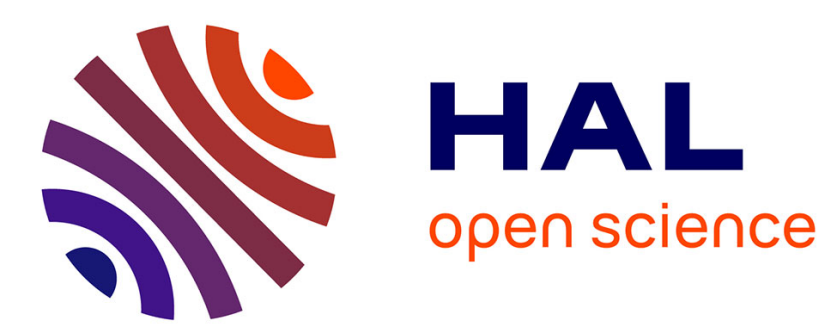

\title{
Fast-axis orientation dependence on driving voltage for a Stokes polarimeter based on concrete liquid-crystal variable retarders
}

\author{
Patrick Terrier, Jean-Michel Charbois, Vincent Devlaminck
}

\section{- To cite this version:}

Patrick Terrier, Jean-Michel Charbois, Vincent Devlaminck. Fast-axis orientation dependence on driving voltage for a Stokes polarimeter based on concrete liquid-crystal variable retarders. Applied optics, 2010, 49 (22), pp.4278-4283. hal-00648809

\section{HAL Id: hal-00648809 \\ https://hal.science/hal-00648809}

Submitted on 9 Mar 2012

HAL is a multi-disciplinary open access archive for the deposit and dissemination of scientific research documents, whether they are published or not. The documents may come from teaching and research institutions in France or abroad, or from public or private research centers.
L'archive ouverte pluridisciplinaire HAL, est destinée au dépôt et à la diffusion de documents scientifiques de niveau recherche, publiés ou non, émanant des établissements d'enseignement et de recherche français ou étrangers, des laboratoires publics ou privés. 


\title{
Fast axis orientation dependence on driving voltage for a
}

\section{Stokes polarimeter based on concrete liquid crystal variable}

\author{
retarders
}

\author{
P. Terrier, J. M. Charbois, V. Devlaminck
}

Univ Lille Nord de France, F-59000 Lille, France

LAGIS, Bâtiment P3

\section{Introduction}

The polarization state of light contains important information about a scene that is complementary to information provided by the light's intensity and color[1]. A polarimetric camera measures the polarization state of the light incident upon the camera. Its usual output is a set of Stokes parameters images. Polarimetric parameters of the reflected light, like direction or degree of polarization, may be derived from these images and provide important information about the observed objects e.g. surface orientation and nature of the material[2][3]. Consequently polarimetric imagery has been found useful in applications as scene analysis, robotics, automatic target recognition[4] or industrial control[5].

Usually the measurement device is a passive[6] or active[7] imaging system (an active system uses an artificial light source to illuminate the scene) that measures the Stokes vector at each spatial location in a scene. A variable retardance scheme is used and the polarimetric modulation is performed by two liquid-crystal variable retarders (LCVRs). Because of the high switching speed of these devices, Stokes parameters images can be acquired and formed at a standard video rate. The LCVRs are key components of the system, and many authors have characterized their performance in terms of precision of modulation and image quality[8][9]. Several authors have demonstrated that 
the optical retardance of LCVR changes strongly with temperature [9][10]. Therefore a temperature control system is often used for precise measurements. In this paper we show that fast axis orientation may as well be dependent on applied voltage. We address this problem and demonstrate that the consideration of this phenomenon improves the mathematical model allowing to measure more precisely the Stokes parameters.

In Section 2, we describe the Stokes imaging system and evaluate the imperfection of the LCVRs polarimetric theoretical model. In section 3, we illustrate the improvement of the algorithms when a rotation on the LCVR fast axis is taken into account in the model.

\section{Stokes Imaging System}

The Stokes polarimeter device is based on the use of two Meadowlark Optics LRC-300 LCVRs. Each of these optical components makes it possible to modify the polarization state of the incident light wave without requiring mechanical actions on the device (rotations). The Stokes parameters of the incident light, $\mathbf{S}=\left[S_{0} S_{1} S_{2} S_{3}\right]^{T}$ ( $T$ denotes the transpose of the vector), can thus be estimated precisely and rapidly[11]. The variable parameter of the device is a pair of retardances $\left(\delta_{1}, \delta_{2}\right)$ adjusted by the amplitude of a square alternative voltage that is applied to the LCVRs through an intermediately located command interface board. First we describe the operation of the Stokes polarimeter, and then we characterize the polarimetric response of the LCVRs.

\section{A. Operation of the Stokes Imaging Polarimeter}

All the experiments presented in this paper are made with a quasi-monochromatic non coherent light source, centred on wavelength $\lambda_{0}=640 \mathrm{~nm}$ (red LED). We have described, in a previous paper[4], the Stokes imaging polarimeter. We remind that the analysed light wave passes successively through two variable retarders and a linear polarizer. The resultant light intensity is then measured by a CCD camera. The system components are shown in Fig. 1. 


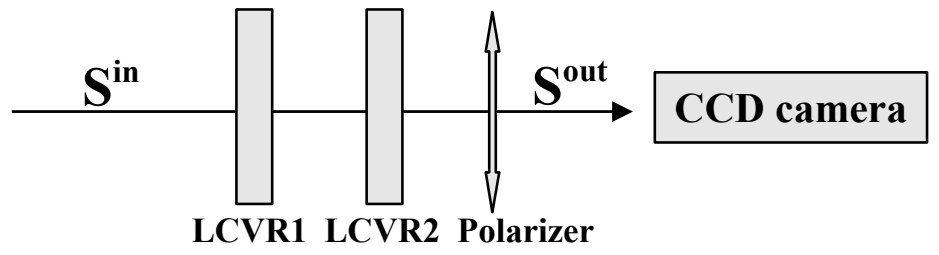

Fig. 1: System components.

The influence of each of these optical components on the state of polarization of the light wave is modelled by a Stokes-Mueller formalism. The Stokes vector $\mathbf{S}^{\text {out }}$ of the light wave at the polarizer output is related to input Stokes vector $\mathbf{S}^{\text {in }}$ by Equation(1):

$$
\mathbf{S}^{\text {out }}=M_{P O L} M_{R}\left(\delta_{2}\right) M_{R}\left(\delta_{1}\right) \mathbf{S}^{\text {in }}=M_{G L O B A L} \mathbf{S}^{\text {in }}
$$

where $M_{R}(\delta)$ is the Mueller matrix of a pure retarder with retardance $\delta$ and $M_{P O L}$ is the Mueller matrix of a linear polarizer. For a given pair of retardances $\left(\delta_{1}, \delta_{2}\right)$, light intensity $I_{i}$ measured by the CCD camera corresponds to Stokes parameter $S_{0}^{\text {out }}$. It is thus a linear function, given by Eq. (2), of the input Stokes parameters $\mathbf{S}^{\text {in }}$ of the observed light:

$$
I=S_{0}^{\text {out }}=A\left(\delta_{1}, \delta_{2}\right) S_{0}^{i n}+B\left(\delta_{1}, \delta_{2}\right) S_{1}^{i n}+C\left(\delta_{1}, \delta_{2}\right) S_{2}^{i n}+D\left(\delta_{1}, \delta_{2}\right) S_{3}^{i n}
$$

where the parameters $[\mathrm{A}, \mathrm{B}, \mathrm{C}, \mathrm{D}]$ correspond to the first line of $M_{G L O B A L}$ matrix (Eq. (1)) . As a consequence, for each pixel of the image of the observed scene, one can estimate the four parameters of Stokes vector $\mathbf{S}^{\text {in }}$ by carrying out $\mathrm{N}$ acquisitions $\mathbf{I}=\left\{I_{i}, i \in[1, N]\right\}$ for $N$ combinations of retardances $\left(\delta_{1}, \delta_{2}\right)$ : 


$$
\begin{gathered}
\mathbf{I}=M_{\text {LIGHT }} \mathbf{S}^{\text {in } \Leftrightarrow}\left(\begin{array}{c}
I_{1} \\
I_{2} \\
\vdots \\
I_{N}
\end{array}\right)=\left[\begin{array}{cccc}
A_{1} & B_{1} & C_{1} & D_{1} \\
A_{2} & B_{2} & C_{2} & D_{2} \\
\vdots & \vdots & \vdots & \vdots \\
A_{N} & B_{N} & C_{N} & D_{N}
\end{array}\right]\left(\begin{array}{c}
S_{0} \\
S_{1} \\
S_{2} \\
S_{3}
\end{array}\right) \\
\mathbf{S}^{\text {in }}=M_{\text {LIGHT }}^{-1} \mathbf{I}
\end{gathered}
$$

where matrix $M_{L I G H T}$ is a function of $\mathrm{N}$ couples of retardances (one couple per line) and of the positioning of the optical components.

Several authors[12][13] have studied the design of the Stokes polarimeter device in order to optimize the accuracy of the estimated Stokes parameter $\mathbf{S}^{\text {in }}$. They have proposed to use sequentially four pairs of retardation values $\left(\delta_{1}, \delta_{2}\right)$ for each LCVR. These values are chosen in order to minimize the condition number of the resultant square matrix $M_{\text {LIGHT }}$ (the ratio of the largest singular value of the matrix over the smallest one). Calculations exhibit several global minima, all equal to $\sqrt{3}$. Some of these minima are reached for a retardation sequence of the form:

$$
\left(\delta_{1}, \delta_{2}\right)=\left(\Delta_{1} \Delta_{1}\right),\left(\Delta_{1} \Delta_{2}\right),\left(\Delta_{2} \Delta_{1}\right),\left(\Delta_{2} \Delta_{2}\right)
$$

with $\Delta_{1}=135^{\circ}, \Delta_{2}=315^{\circ}$ and for LCVRs orientations given by $\theta_{1}$ (orientation of the first $\mathrm{LCVR})=72.4^{\circ}$ and $\theta_{2}$ (orientation of the second one) $=27.4^{\circ}$.

In order to obtain an accurate estimate of the observed Stokes parameters $\mathbf{S}^{\text {in }}$, we have to know precisely the parametric form of matrix $M_{R}(\delta)$ and $M_{P O L}$ and to adjust the different parameters $\Delta_{1}$, $\Delta_{2}, \theta_{1}$ and $\theta_{2}$ to their optimal values. First, it is necessary to calibrate the LCVRs, that means to find the driving voltages allowing to tune these optimal retardation values. 


\section{B. Calibration of the liquid-crystal variable retarders}

For the calibration of LCVR we place it between two linear polarizers. The first one generates a vertically polarized light wave that is retarded by the liquid crystal variable retarder. After being retarded by the liquid crystal, the light is analysed by the second polarizer which moves on a rotation stage driven by a stepper motor controller (resolution=2.13 $10^{-4}$ degrees). Finally the intensity of the light is measured by a 12 bit CCD camera. The Stokes vector $\mathbf{S}$ " at the second polarizer output is obtained by the Mueller formalism:

$$
\mathbf{S}^{\prime \prime}=M_{P O L} M_{R}(\delta) \mathbf{S}^{\prime}
$$

where $\mathbf{S}^{\prime}=[1100]^{T}$ represents the light linearly polarized by the first polarizer and:

$M_{R}(\delta)=\left[\begin{array}{cccc}1 & 0 & 0 & 0 \\ 0 & \cos ^{2} 2 \phi+\sin ^{2} 2 \phi \cos \delta & \sin 2 \phi \cos 2 \phi(1-\cos \delta) & -\sin 2 \phi \sin \delta \\ 0 & \sin 2 \phi \cos 2 \phi(1-\cos \delta) & \sin ^{2} 2 \phi+\cos ^{2} 2 \phi \cos \delta & \cos 2 \phi \sin \delta \\ 0 & \sin 2 \phi \sin \delta & -\cos 2 \phi \sin \delta & \cos \delta\end{array}\right]$

is the Mueller matrix for the liquid crystal where the retardation is $\delta$ and the fast axis orientation is

$\Phi$. The light at the LCVR output is analysed by the second polarizer which can be described by Eq. (8):

$$
M_{P O L}=\frac{1}{2}\left[\begin{array}{cccc}
1 & \cos 2 \theta & \sin 2 \theta & 0 \\
\cos 2 \theta & \cos ^{2} 2 \theta & \sin 2 \theta \cos 2 \theta & 0 \\
\sin 2 \theta & \sin 2 \theta \cos 2 \theta & \sin ^{2} 2 \theta & 0 \\
0 & 0 & 0 & 0
\end{array}\right]
$$

where $\theta$ is the orientation angle of the polarizer.

Using these matrices, the intensity $S_{0}^{\prime \prime}$ measured by the camera at the second polarizer output can be obtained by Eq. (9).

$S_{0}^{\prime \prime}=\frac{1}{2}\left[1+\cos 2 \theta\left(\cos ^{2} 2 \phi+\sin ^{2} 2 \phi \cos \delta\right)+\sin 2 \theta \sin 2 \phi \cos 2 \phi(1-\cos \delta)\right]$ 
Thus, when we set $\Phi=45^{\circ}, S_{0}^{\prime \prime}$ becomes:

$$
S_{0}^{\prime \prime}=\frac{1}{2}[1+\cos 2 \theta \cos \delta]
$$

Therefore, if we measure the intensity ${ }^{\prime \prime}$ for multiple angles $\theta$ by rotating the second polarizer, we can estimate the retardation $\delta$ of the LCVR. The procedure is:

- Set the LCVR between polarizers and rotate the second polarizer to check the variation of the intensity. The orientation of the LCVR is adjusted so as to measure the same sinusoidal intensity variation as in the case of no LCVR. Thus the fast axis of the LCVR is set to be parallel to the axis of the first polarizer $\left(\Phi=0^{\circ}\right)$.

- Rotate the LCVR by $45^{\circ}$.

- Put the voltage on the LCVR and start the measurement by rotating the second polarizer.

- Determine the retardation using Equation (10).

With this procedure we derive the retardations for each voltage. We have done this calibration for LCVR1 and LCVR2 of the polarimeter (see Fig. 1). One of the results is shown in Fig. 2 where the temperature of the $\mathrm{LCVR}$ is $30^{\circ} \mathrm{C}$.

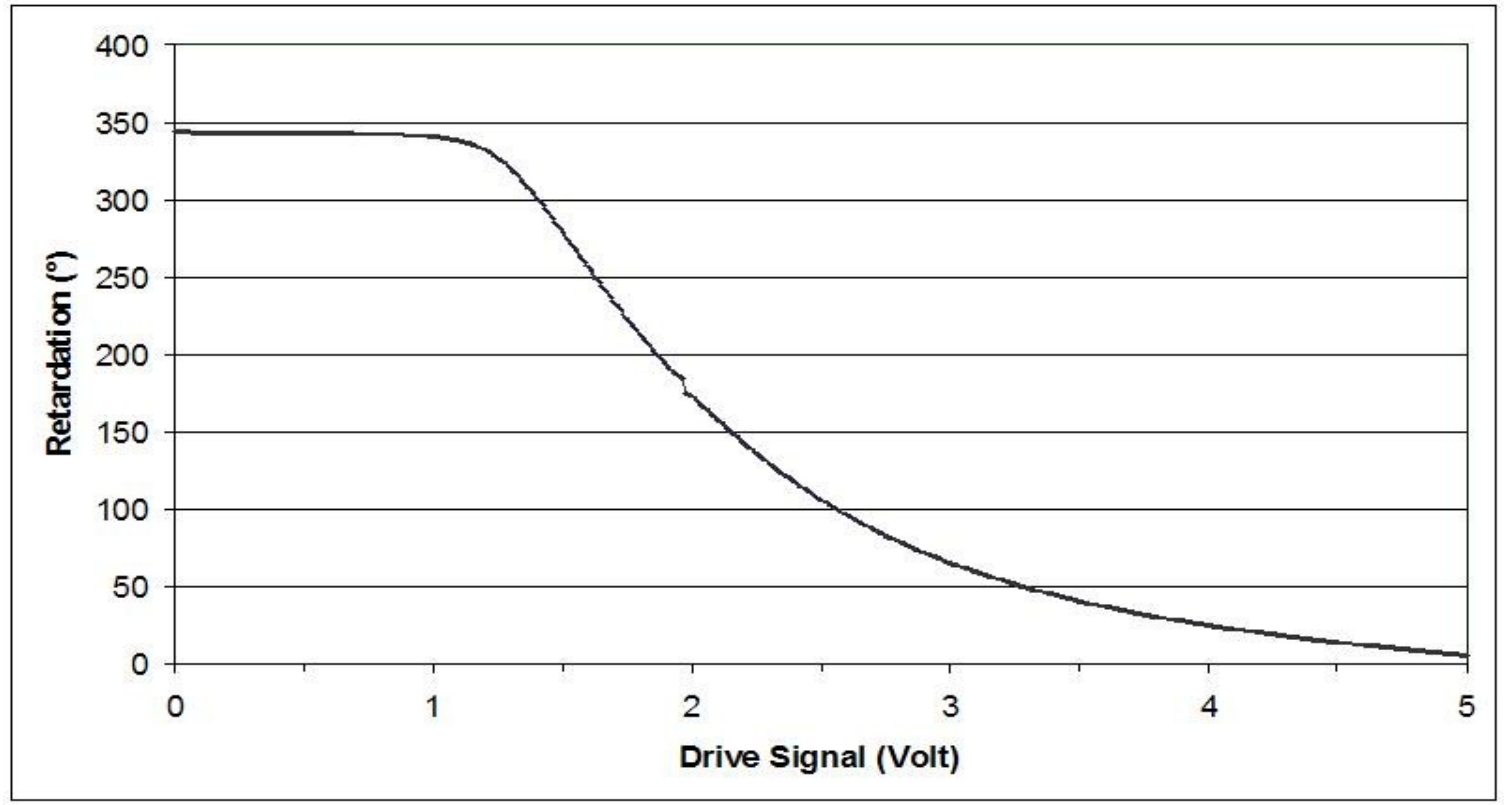

Fig. 2: LCVR1 retardation versus voltage. 
According to this curve we determine the voltages $\mathrm{V}_{1}$ and $\mathrm{V}_{2}$ that allow us to adjust respectively the retardations to the optimal values $\Delta_{1}=135^{\circ}, \Delta_{2}=315^{\circ}$ (minimal condition number of the matrix $\left.M_{\text {LIGHT }}[12]\right)$. We found $\left(\mathrm{V}_{11}, \mathrm{~V}_{21}\right)=(2.280 \mathrm{~V}, 1.391 \mathrm{~V})$ for LCVR1 and $\left(\mathrm{V}_{12}, \mathrm{~V}_{22}\right)=(2.217 \mathrm{~V}, 1.416 \mathrm{~V})$ for LCVR2.

\section{Imperfection of the LCVR polarimetric theoretical model}

By driving the LCVRs with optimal voltages $(\mathrm{V} 1, \mathrm{~V} 2)$ we acquire four images $\mathbf{I}=\left\{I_{i}, i \in[1,4]\right\}$ of the observed scene for four combinations of the driving voltage:

$$
\left(V_{1}, V_{2}\right)=\left(V_{11} V_{12}\right),\left(V_{11} V_{22}\right),\left(V_{21} V_{12}\right),\left(V_{21} V_{22}\right)
$$

and form the matrix $M_{L I G H T}$ according to Eq. (3).

The matrix (called theoretical matrix $M_{\text {LIGHT-THEO }}$ ) obtained using the theoretical Mueller models of the optical components is given by:

$$
M_{\text {LIGHT-THEO }}=\left[\begin{array}{cccc}
0.5000 & -0.4713 & 0.1669 & -0.0005 \\
0.5000 & 0.2364 & 0.1661 & 0.4081 \\
0.5000 & -0.0008 & -0.5000 & 0.0005 \\
0.5000 & 0.2358 & 0.1669 & -0.4081
\end{array}\right]
$$

and has a condition number equal 1.735 (very close to optimal value $\sqrt{3}$ [12]). We remind that the condition number is defined by the ratio of the largest singular value of the matrix over the smallest one.

We have measured experimentally the Mueller matrix $M_{G L O B A L}$ for the four combinations of the driving voltage [14]. Both the polarization state analyzer and generator of the Mueller matrix polarimeter are based on a fixed linear polarizer and a rotated wave plate. With these four matrices we form the experimental matrix $M_{\text {LIGHT-EXP }}$ that generates the four images $\boldsymbol{I}$. We have obtained: 


$$
M_{\text {LIGHT-EXP }}=\left[\begin{array}{cccc}
0.5001 & -0.4928 & 0.1429 & 0.0382 \\
0.5003 & 0.1514 & 0.2302 & 0.4218 \\
0.4994 & 0.0907 & -0.4962 & -0.0870 \\
0.5001 & 0.2273 & 0.1649 & -0.4188
\end{array}\right]
$$

The condition number of this measured experimental matrix is 1.914 and is thus not as good as the expected theoretical value. Furthermore these matrices are slightly different. In order to compare them we use the norm of the difference between $M_{\text {LIGHT-THEO }}$ and $M_{\text {LIGHT-EXP }}$ (the largest singular value of the difference) and find 0.1546 .

We can derive two consequences from these results. On the one hand if the theoretical matrix $M_{\text {LIGHT-THEO }}$ is used during the inversion process (see Eq. (4)) the Stokes vectors $\mathbf{S}^{\text {in }}$ are misestimated, since the polarization images $\boldsymbol{I}$ are generated from the matrix $M_{\text {LIGHT-EXP. }}$ On the other hand, though the theoretical optimal settings are used (voltages (V1,V2) and orientations $\left(\theta_{1}, \theta_{2}\right)$ ) we do not find the expected matrix with optimal condition number. Thus performing the inversion process with matrix $M_{\text {LIGHTEXP }}$ is noise sensitive.

\section{Improvement of the Stokes polarimeter}

\section{A. Adjustment of the LCVR Mueller matrix}

The difference between the expected theoretical matrix $M_{\text {LIGHT-THEO }}$ and the experimental measured matrix $M_{L I G H T-E X P}$ is essentially due to the fact that the model of the LCVR Mueller matrix (Eq. (7)) is only an approximation. It is worth noticing that the angular position $\Phi$ of the LCVR fast axis is supposed to be voltage independent in this model. Only the retardation $\delta$ fluctuates according to the driving voltage V. However, in practice, the position of the axis fluctuates slightly according to the voltage as we can see in the following experiment:

- We place the LCVR component between two polarizers. The first one is fixed in a 
reference position and we rotate the second one to check the variation of the intensity. No voltage is applied and the orientation of the LCVR is adjusted so as to measure the same intensity variation as in the case of no LCVR (that is to say a sinusoidal variation) shown in Fig. 3. Thus the fast axis of the LCVR is set to be parallel to the axis of the first polarizer $\left(\Phi=0^{\circ}\right)$.

- We do not move the LCVR and apply a driving voltage (V=2 volts for example in this experiment). The second polarizer is rotated. As we can see the intensity variation is sinusoidal again but the measured sinusoid is shifted with regard to the reference sinusoid.

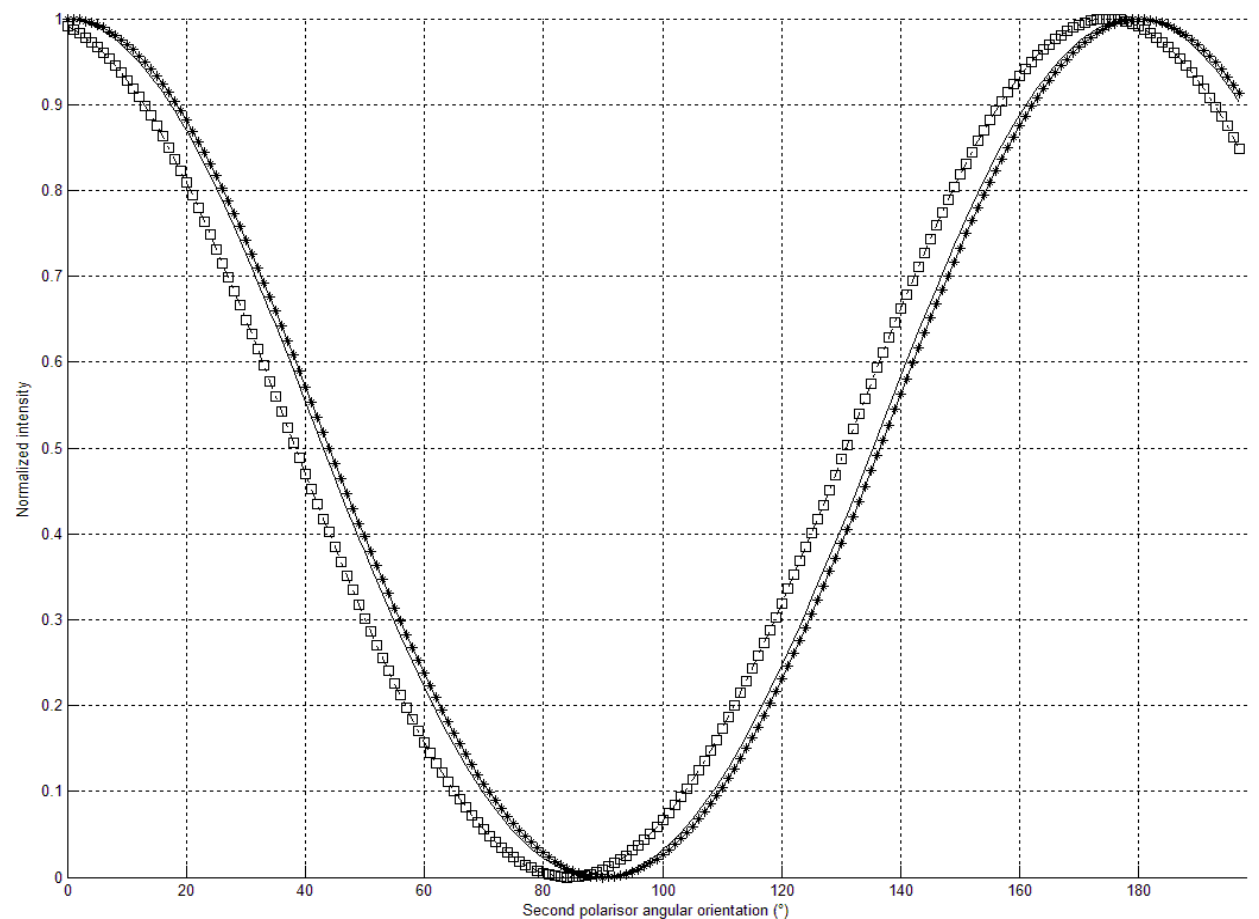

Fig. 3: Variation of the intensity with the rotation of the second polarizer: The solid curve is the reference sinusoid (without LCVR), the + show the measured values without driving voltage, the squares show the measurements for $\mathrm{V}=2$ volts 
This shift on the intensity fluctuation can be modelled by a variation of the position of the LCVR fast axis when a driving voltage is applied.

For LCVR1 and 2, we have measured the difference between the reference position (without driving signal) and the actual position when voltage is applied. This difference for LCVR1 is plotted versus the driving voltage in Fig. 4.

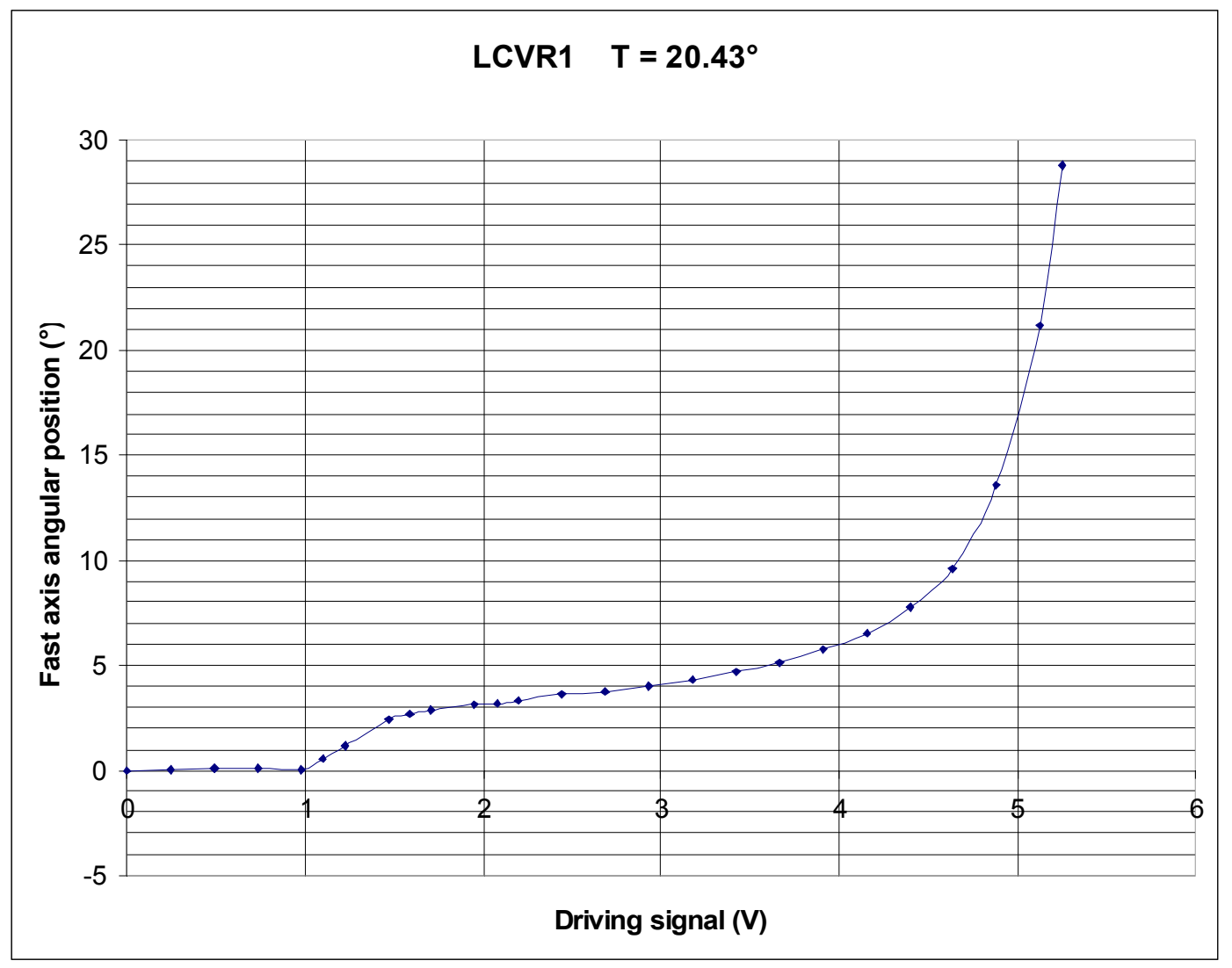

Fig. 4: Fast axis angular position versus driving voltage for LCVR 1

In order to improve the matrix $M_{\text {LIGHT-THEO }}$ (minimization of the difference between $M_{\text {LIGHT-THEO }}$ and $\left.M_{\text {LIGHT-EXP }}\right)$ that is used during the inversion process we have to take into account this phenomenon. We remind that we use two pairs of voltage to minimize the matrix condition number $\left(\mathrm{V}_{11}, \mathrm{~V}_{21}\right)=(2.280 \mathrm{~V}, 1.391 \mathrm{~V})$ for LCVR1 and $\left(\mathrm{V}_{12}, \mathrm{~V}_{22}\right)=(2.217 \mathrm{~V}, 1.416 \mathrm{~V})$ for LCVR2. For each of these voltages we measure the drift $\Delta \Phi$ of the LCVR fast axis position. We find respectively $\Delta \Phi=\left(3.5^{\circ}, 5^{\circ}\right)$ for $\operatorname{LCVR} 1$ and $\Delta \Phi=\left(3^{\circ}, 2^{\circ}\right)$ for LCVR 2 
Taking into account these drifts in matrix $M_{\text {LIGHT-THEO }}$ we obtain $M_{\text {LIGHT-THEO-ADJ }}$ given by Eq. (14):

$$
M_{\text {LIGHT-THEO-ADJ }}=\left[\begin{array}{cccc}
0.5000 & -0.4732 & 0.1518 & 0.0555 \\
0.5000 & 0.1649 & 0.2265 & 0.4141 \\
0.5000 & 0.0840 & -0.4890 & -0.0617 \\
0.5000 & 0.2555 & 0.1375 & -0.4072
\end{array}\right]
$$

The difference between this adjusted matrix $M_{\text {LIGHT-THEO-ADJ }}$ and the experimental matrix $M_{\text {LIGHT-EXP }}$ is 0.0447 (norm of the difference) by comparison with the previous difference between $M_{\text {LIGHT-THEO }}$ and $M_{\text {LIGHTEXP }}$ that was 0.1546 . Consequently these matrices are closer and the precision of estimation on the observed Stokes parameters $\mathbf{S}^{\text {in, }}$ is thus improved.

\section{B. Stokes measurements results}

In order to verify the reliability of the Stokes polarimeter described in section A, two examples for test measurements of well-defined polarimetric waves are shown: the first one is a linearly polarized wave and the second one is a circular polarimetric wave.

- In the first experiment we have observed a monochromatic wave passing through a vertical linear polarizer. The theoretical Stokes vector of this wave is $\mathbf{S}=\left[\begin{array}{llll}1 & -1 & 0 & 0\end{array}\right]^{\mathrm{T}}$. First, we have acquired the four images $\boldsymbol{I}$ and have used the theoretical matrix $M_{\text {LIGHT-THEO }}$ in the inversion process. The estimated normalized Stokes vector is $\mathbf{S}_{\mathbf{T H E O}}=\left[\begin{array}{llll}1 & -0.94 & 0.15 & 0.075\end{array}\right]^{\mathrm{T}}$. The difference between the actual and the estimated vector is 0.1781 (L2 norm). The estimated linear $\left(\sqrt{S_{1}^{2}+S_{2}^{2}}\right)$ and circular $\left(\sqrt{S_{3}^{2}}\right)$ degrees of polarization are respectively 0.948 and 0.075 (to compare to the theoretical values 1 and 0 ). Then, we have used the refined matrix $M_{\text {LIGHT-THEO-ADJ }}$ to perform the inversion process. In this case the estimated Stokes vector is $\mathbf{S}_{\text {THEO-ADJ }}=\left[\begin{array}{llll}1 & -0.96 & 0.00 & -0.03\end{array}\right]^{\mathrm{T}}$. The distance between actual and estimated Stokes vector 
becomes 0.05 . We can also notice an improvement on the linear and circular degree of polarization: respectively 0.957 and 0.034 .

- In a second experiment we have generated a circular polarimetric wave. For this we place a quarter wave plate, with its fast axis oriented at $45^{\circ}$, behind a vertical linear polarizer. The theoretical Stokes vector of the circular resultant wave is $\mathbf{S}=\left[\begin{array}{llll}1 & 0 & 0 & -1\end{array}\right]^{\mathrm{T}}$. As in the first experiment we have acquired the four images $\boldsymbol{I}$ and used the theoretical matrix $M_{\text {LIGHT-THEO }}$ in the inversion process. The result is $\mathbf{S}_{\mathbf{T H E O}}=\left[\begin{array}{llll}1 & -0.03 & 0.13 & 1.01\end{array}\right]^{\mathrm{T}}$. The difference between estimated and actual vector is equal to 0.1338 . Using the refined matrix $M_{L I G H T-T H E O-A D J}$ the difference becomes 0.052 with an estimated Stokes vector $\mathbf{S}_{\mathbf{T H E O}-\mathbf{A D J}}=\left[\begin{array}{llll}1 & 0.05 & 0.01 & 1.01\end{array}\right]^{\mathrm{T}}$. The estimated linear and circular degree of polarization are respectively 0.133 and 1.013 for the inversion with matrix $M_{\text {LIGHT-THEO }}$. With the refined matrix these parameters become respectively 0.047 and 1.012 . We can notice the improvement of the estimation of the linear degree of polarisation.

\section{Discussion and summary}

Using LCVRs, errors caused by moving parts, unavoidable when using mechanical rotation [15] (inertia with its acceleration and braketimes, misalignments of the signal on the detector,...), are eliminated. Precise rotation stages, stepper motors,... are dispensable. On the other hand, the main well known disadvantage is that the retardation introduced by LCVR depends on temperature. To take this into account, several authors recommend to calibrate the component just before carrying out measurements and calibrate it again every 10 minutes [16]. During this time,changes in the plot voltage retardation were not found.

In the presented work we show that the most often used theoretical model for LCVR Mueller matrix may be just regarded as an approximation. It is possible to refine this matrix model by introducing a fast axis position dependent on the driving voltage. The experimental matrix that generates intensity 
and the theoretical matrix used to estimate the observed Stokes vector, are thus closer. Consequently, the precision of estimation on the observed Stokes parameters $\mathbf{S}^{\text {in }}$ is improved.

Taking into account this refine model to derive an optimal setting, leading to an optimal condition number, is under investigation.

\section{Acknowledgements}

We would like to thank Prof. Laurent Bigue of the MIPS laboratory, Université de Haute Alsace, for useful discussions and suggestions. 


\section{References}

1. W. A. Shurcliff, Polarized light, (Harvard University Press, Harvard, 1962)

2. L. B. Wolff and T. E. Boult, "Constraining object features using a polarization reflectance model", IEEE Trans. Pattern Anal. Mach. Intell. 13, 635-657 (1991)

3. D. Miyazaki, R. T. Tan, K. Hara, and K. Ikeuchi, "polarization-based inverse rendering from a single view", in Proc. IEEE Conference on Computer Vision, 982-987 (2003)

4. F. Goudail, P. Terrier, Y. Takakura, L. Bigué, F. Galland, and V. DeVlaminck , "Target detection with a liquid-crystal-based passive Stokes polarimeter", Appl. Opt. 43, 274-282 (2004)

5. Patrick Terrier, Vincent Devlaminck, and Jean Michel Charbois, "Segmentation of rough surfaces using a polarization imaging system", J. Opt. Soc. Am. A 25, 423-430 (2008)

6. J. Scott Tyo, Dennis L. Goldstein, David B. Chenault, and Joseph A. Shaw, "Review of passive imaging polarimetry for remote sensing applications", Appl. Opt. 45, 5453-5469 (2006)

7. Olivier Morel, Christophe Stolz, Fabrice Meriaudeau, and Patrick Gorria, "Active lighting applied to three-dimensional reconstruction of specular metallic surfaces by polarization imaging", Appl. Opt. 45, $4062-4068$ (2006)

8. J. E. Wolfe, R. A. Chipman, "Polarimetric characterization of liquid-crystal-on-silicon panels", Appl. Opt. 45, 1688-1703 (2006)

9. J. S. Baba and P. R. Boudreaux , "Wavelength, temperature, and voltage dependent calibration of a nematic liquid crystal multispectral polarization generating device", Appl. Opt. 46, 5539-5544 (2007)

10. R. L. Heredero, N. Uribe-Patarroyo, T. Belenguer, G. Ramos, A. Sánchez, M. Reina, V. Martínez Pillet, and A. Álvarez-Herrero , "Liquid-crystal variable retarders for aerospace polarimetry applications", Appl. Opt. 46, 689-698 (2007)

11. B. Laude-Boulesteix, A. De Martino, B. Drévillon, L. Schwartz, "Mueller Polarimetric Imaging System with Liquid Crystals", Appl. Opt. 43, 2824-2832 (2004)

12. J. S. Tyo, "Noise equalization in Stokes parameter obtained by use of variable-retardance polarimeters", Opt. Lett. 25, 1198-1200 (2000)

13. A. De Martino, Y. Kim, E. Garcia-Caurel, B. Laude and B. Drevillon, "Optimized mueller polarimeter with liquid crystals", Opt. Lett. 28, 616-618 (2003) 
14. Eric Compain, Stéphane Poirier, and Bernard Drevillon, "General and Self-Consistent Method for the Calibration of Polarization Modulators, Polarimeters, and Mueller-Matrix Ellipsometers", Appl. Opt. 38, 3490-3502 (1999)

15. Jawad Elsayed Ahmad and Yoshitate Takakura, "Error analysis for rotating active Stokes-Mueller imaging polarimeters", Opt. Lett. 31, 2858-2860 (2006)

16. J. M. Bueno, "Polarimetry using liquid-crystal variable retarders: theory and calibration", J. Opt. A: Pure Appl. Opt. 2, 216-222 (2000) 